\title{
ITERATED INTEGRAL TRANSFORMS OF CARATHEODORY FUNCTIONS AND THEIR APPLICATIONS TO ANALYTIC AND UNIVALENT FUNCTIONS
}

\author{
K. O. BABALOLA AND T. O. OPOOLA
}

\begin{abstract}
In this paper we develop and study some integral transforms of Caratheodory functions. We apply the transforms to study certain other classes of analytic and univalent functions both to obtain new results and provide new proofs of some known ones.
\end{abstract}

\section{Introduction}

Let $C$ be the complex plane. Denote by $P$ the class of functions:

$$
p(z)=1+p_{1} z+p_{2} z^{2}+\cdots
$$

which are analytic in the unit disk $E=\{z:|z|<1\}$ and satisfy $\operatorname{Re} p(z)>0, z \in E$.

The family $P$, known as the Caratheodory functions, plays a vital role in geometric function theory. That is so because many analytic and univalent functions have representation in terms of functions in it. For instance, the normalized analytic function:

$$
f(z)=z+a_{2} z^{2}+\cdots
$$

is said to be starlike, convex, or belongs to the classes $S_{0}, R$, and $B_{1}(\alpha)$, provided the geometric quantities $z f^{\prime}(z) / f(z), 1+z f^{\prime \prime}(z) / f^{\prime}(z), f(z) / z, f^{\prime}(z)$ and $f(z)^{\alpha-1} f^{\prime}(z) / z^{\alpha-1}$, (where $\alpha>0$ is real) respectively belong to the family $P[4,7,8,9,10]$.

The object of the present paper is to identify certain iterated integral transforms of functions in the class $P$, which have arisen from the study of the classes of functions defined in $[1,6]$. We relate the transforms with those classes and give some of their applications in the latter sections. We begin with

Definition 1.1. Let $p \in P$ and $\alpha>0$ be real. The $n$th iterated integral transform of $p(z), z \in E$ is defined as

$$
p_{n}(z)=\frac{\alpha}{z^{\alpha}} \int_{0}^{z} t^{\alpha-1} p_{n-1}(t) d t, \quad n \in N
$$

Received August 2, 2005.

2000 Mathematics Subject Classification. 30C45.

Key words and phrases. Iterated integral transform, Caratheodory function, analytic and univalent function. 
with $p_{0}(z)=p(z)$.

Note that since $p_{0}(z)$ belongs to $P$, the transform $p_{n}(z), n \in N$ is analytic, and $p_{n}(0)=1$ and $p_{n}(z) \neq 0$ for $z \in E$.

We shall denote the family of the $n$th iterated integral transform of $p \in P$ by $P_{n}$. With the above definition, it is easy to see that if $p(z)$ is given by $(1.1)$, then

$$
p_{n}(z)=1+\sum_{k=1}^{\infty} p_{n, k} z^{k}
$$

where

$$
p_{n, k}=\frac{\alpha^{n}}{(\alpha+k)^{n}} p_{k}, \quad k=1,2, \ldots
$$

and we remark here that an analogue of the well-known Caratheodory lemma for the $p_{n}(z)$ is the inequality:

$$
\left|p_{n, k}\right| \leq \frac{2 \alpha^{n}}{(\alpha+k)^{n}}, \quad k=1,2, \ldots
$$

By setting $p_{0}(z)=L_{0}(z)=(1+z) /(1-z)$ we see easily that the $n$th iterated integral transform of the Mobius function is:

$$
L_{n}(z)=\frac{\alpha}{z^{\alpha}} \int_{0}^{z} t^{\alpha-1} L_{n-1}(t) d t, \quad n \in N .
$$

The function $L_{n}(z)$ plays a central role in the family $P_{n}$ similar to role of the Mobius function $L_{0}(z)$ in the family $P$.

Remark 1.2. Let us denote $p_{n}(z)$ by $\chi_{n}^{(\alpha)}(p(z))$. Then for any $p \in P$ and $m, n \in N_{0}$, we have

$$
\chi_{m}^{(v)}\left(\chi_{n}^{(\alpha)}(p(z))\right)=\chi_{n}^{(\alpha)}\left(\chi_{m}^{(v)}(p(z))\right)
$$

where $v>0$ is also real. This is easily seen using (1.4) and (1.5). Now for $v=\alpha,(1.8)$ gives

$$
\chi_{m}^{(\alpha)}\left(\chi_{n}^{(\alpha)}(p(z))\right)=\chi_{n}^{(\alpha)}\left(\chi_{m}^{(\alpha)}(p(z))\right)=\chi_{n+m}{ }^{(\alpha)}(p(z)) .
$$

In Section 2 we provide a lemma, which we shall depend on in the study of functions in the family $P_{n}$ in Section 3. Some applications of the integral transforms in analytic and univalent functions theory are mentioned in Section 4.

\section{A Fundamental Lemma}

In order to be able to adequately discuss the transformation $p_{n}(z)$, we require some preliminary concepts.

Definition 2.1. Let $u=u_{1}+u_{2} i, v=v_{1}+v_{2} i$ and $\gamma \neq 1$ be a nonnegative real number. Define $\Psi_{\gamma}$ as the set of functions $\psi(u, v): C \times C \rightarrow C$ satisfying:

(a) $\psi(u, v)$ is continuous in a domain $\Omega$ of $C \times C$. 
(b) $(1,0) \in \Omega$ and $\operatorname{Re} \psi(1,0)>0$.

(c) $\operatorname{Re} \psi\left(\gamma+(1-\gamma) u_{2} i, v_{1}\right) \leq \gamma$ when $\left(\gamma+(1-\gamma) u_{2} i, v_{1}\right)$ is a point of the domain $\Omega$ and $2 v_{1} \leq-(1-\gamma)\left(1+u_{2}^{2}\right)$ for $0 \leq \gamma<1$.

(d) $\operatorname{Re} \psi\left(\gamma+(1-\gamma) u_{2} i, v_{1}\right) \geq \gamma$ when $\left(\gamma+(1-\gamma) u_{2} i, v_{1}\right)$ is a point of the domain $\Omega$ and $2 v_{1} \geq(\gamma-1)\left(1+u_{2}^{2}\right)$ for $\gamma>1$.

The set $\Psi_{\gamma}$ is nonempty. The following examples of such functions $\psi(u, v)$ belong to the set.

(i) $\psi_{1}(u, v)=u+v / \alpha$, $\operatorname{Re} \alpha>0$ and $\Omega=C \times C$.

(ii) $\psi_{2}(u, v)=u+\xi v / u, \xi>0$ is real and $\Omega=[C-\{0\}] \times C$.

(iii) $\psi_{3}(u, v)=u+v /(\xi+u), \xi$ is real, $\xi+\gamma>0$ and $\Omega=[C-\{-\xi\}] \times C$.

(iv) $\psi_{4}(u, v)=u e^{v}+v$, with $\Omega=C \times C$.

The set $\Psi_{\gamma}$ is closed under addition, and for any $m>0, m \psi \in \Psi_{\gamma}$ if $\psi \in \Psi_{\gamma}$. If $\gamma=0$, we simply write $\Psi$ in place of $\Psi_{0}$. The set $\Psi$ has been defined in several literatures and is found to contain many more examples $[3,5]$. Furthermore the inequalities $2 v_{1} \leq$ $-(1-\gamma)\left(1+u_{2}^{2}\right)$ and $2 v_{1} \geq(\gamma-1)\left(1+u_{2}^{2}\right)$ may be replaced respectively by $v_{1} \leq 0$ and $v_{1} \geq 0$. These weaker conditions are easier to work with algebraically although some generality may be lost. For instance, $\psi_{5}=u+2 v+\left(1-u^{2}\right) / 2$ requires the original stronger condition in order to belong to the set $\Psi$.

Definition 2.2. Let $\psi \in \Psi_{\gamma}$ with corresponding domain $\Omega$. Define $P\left(\Psi_{\gamma}\right)$ as the set of functions $p(z)$ given as $(p(z)-\gamma) /(1-\gamma)=1+p_{1} z+p_{2} z^{2}+\cdots$ which are regular in $E$ and satisfy:

(i) $\left(p(z), z p^{\prime}(z)\right) \in \Omega$.

(ii) $\operatorname{Re} \psi\left(p(z), z p^{\prime}(z)\right)>\gamma$ when $z \in E$ and $0 \leq \gamma<1$.

(iii) $\operatorname{Re} \psi\left(p(z), z p^{\prime}(z)\right)<\gamma$ when $z \in E$ and $\gamma>1$.

The concepts (ii) and (iii) above are not vacuous since for sufficiently small $\left|p_{1}\right|$ (depending on $\psi$ ), the function $p(z)$ given by $(p(z)-\gamma) /(1-\gamma)=1+p_{1} z$ satisfies them. In particular, let $\psi_{1}(u, v)=u+v$, then $\psi\left(p(z), z p^{\prime}(z)\right)=p(z)+z p^{\prime}(z)=\gamma+(1-\gamma)\left[1+2 p_{1} z\right]$. Thus if $0 \leq \gamma<1$, then $\operatorname{Re} \psi\left(p(z), z p^{\prime}(z)\right) \geq \gamma+(1-\gamma)\left[1-2\left|p_{1}\right||z|\right]>\gamma+(1-\gamma)\left[1-2\left|p_{1}\right|\right]$ so that for sufficiently small $\left|p_{1}\right|$, Re $\psi\left(p(z), z p^{\prime}(z)\right)>\gamma$ whereas for $\gamma>1$, we have Re $\psi\left(p(z), z p^{\prime}(z)\right)=\gamma+(\gamma-1)\left[-2 \operatorname{Re} p_{1} z-1\right] \leq \gamma+(\gamma-1)\left[2\left|p_{1}\right||z|-1\right]<\gamma+(\gamma-1)\left[2\left|p_{1}\right|-1\right]$ hence for sufficiently small $\left|p_{1}\right|$, Re $\psi\left(p(z), z p^{\prime}(z)\right)<\gamma$.

Lemma 2.3. Let $p \in P\left(\Psi_{\gamma}\right)$. Then

$$
\operatorname{Re} p(z)\left\{\begin{array}{l}
>\gamma, \text { if } 0 \leq \gamma<1 \\
<\gamma, \text { if } \gamma>1
\end{array}\right.
$$

Proof. Since $p \in P\left(\Psi_{\gamma}\right), p(z)$ given by $(p(z)-\gamma) /(1-\gamma)=1+p_{1} z+p_{2} z^{2}+\cdots$ is analytic in $E$. If we set

$$
\frac{p(z)-\gamma}{1-\gamma}=\frac{1+w(z)}{1-w(z)}, \quad z \in E
$$


then $w(0)=0, w(z) \neq 1$ and $w(z)$ is meromorphic in $E$. Suppose there exists a point $z_{0} \in E$ such that for $|z| \leq\left|z_{0}\right|, \max |w(z)|=\left|w\left(z_{0}\right)\right|=1$. Then by Jack's Lemma (see $[3])$,

$$
z_{0} w^{\prime}\left(z_{0}\right)=m w\left(z_{0}\right), m \geq 1 .
$$

Since $\left|w\left(z_{0}\right)\right|=1$ and $w\left(z_{0}\right) \neq 1$ we must have

$$
\frac{1+w\left(z_{0}\right)}{1-w\left(z_{0}\right)}=A i, A \text { real }
$$

Thus from (2.1) we get

$$
p\left(z_{0}\right)=\gamma+(1-\gamma) A i
$$

Also from (2.1) we obtain

$$
z p^{\prime}(z)=\frac{2(1-\gamma) z w^{\prime}(z)}{(1-w(z))^{2}}
$$

Using (2.2) and (2.3) in (2.5) we have

$$
z_{0} p^{\prime}\left(z_{0}\right)=-\frac{m(1-\gamma)\left(1+A^{2}\right)}{2}=d \text { real. }
$$

Thus at the point $z=z_{0} \in E$ we have $\operatorname{Re} \psi\left(p\left(z_{0}\right), z_{0} p^{\prime}\left(z_{0}\right)\right)=\operatorname{Re} \psi(\gamma+(1-\gamma) A i, d)$, and $2 d \leq-(1-\gamma)\left(1+A^{2}\right)$ if $0 \leq \gamma<1$ and $2 d \geq(\gamma-1)\left(1+A^{2}\right)$ if $\gamma>1$. Since $\psi \in \Psi_{\gamma}$ the conditions (c) and (d) of Definition 2.1 respectively imply that $\operatorname{Re} \psi\left(p\left(z_{0}\right), z_{0} p^{\prime}\left(z_{0}\right)\right) \leq \gamma$ if $0 \leq \gamma<1$ and $\operatorname{Re} \psi\left(p\left(z_{0}\right), z_{0} p^{\prime}\left(z_{0}\right)\right) \geq \gamma$ if $\gamma>1$. These contradict the fact that $p \in P\left(\Psi_{\gamma}\right)$. Therefore we must have $\operatorname{Re} p(z)>\gamma$ if $0 \leq \gamma<1$ and $\operatorname{Re} p(z)<\gamma$ if $\gamma>1$ for all $z \in E$. This completes the proof.

We note here that the case $\gamma=0$ of the above lemma has been proved in [3] and that the class $P\left(\Psi_{\gamma}\right), 0 \leq \gamma<1$, are subclasses of the family of Caratheodory functions. Now we are in a position to characterize functions in the family $P_{n}$.

\section{Some Properties of the Family $P_{n}$}

Theorem 3.1. Let $\gamma \neq 1$ be a nonnegative real number. Then for each $n \in N$,

$$
\begin{aligned}
& \operatorname{Re} p_{n-1}(z)>\gamma \Rightarrow \operatorname{Re} p_{n}(z)>\gamma \text { for } 0 \leq \gamma<1 \text { and } \\
& \operatorname{Re} p_{n-1}(z)<\gamma \Rightarrow \operatorname{Re} p_{n}(z)<\gamma \text { for } \gamma>1
\end{aligned}
$$

Proof. From (1.3) we have

$$
p_{n}(z)+\frac{z p_{n}^{\prime}(z)}{\alpha}=p_{n-1}(z), \quad n \in N .
$$


Now let $n \in N$ and suppose that the conditions of the theorem are satisfied. Then applying Lemma 2.3 to $\psi_{1}$ we have the implication:

$$
\begin{aligned}
& \operatorname{Re}\left(p_{n}(z)+z p_{n}^{\prime}(z) / \alpha\right)>\gamma \Rightarrow \operatorname{Re} p_{n}(z)>\gamma, 0 \leq \gamma<1 \\
& \operatorname{Re}\left(p_{n}(z)+z p_{n}^{\prime}(z) / \alpha\right)<\gamma \Rightarrow \operatorname{Re} p_{n}(z)<\gamma, \gamma>1
\end{aligned}
$$

That is $\operatorname{Re} p_{n-1}(z)>\gamma \Rightarrow \operatorname{Re} p_{n}(z)>\gamma$ for $0 \leq \gamma<1$ and $\operatorname{Re} p_{n-1}(z)<\gamma \Rightarrow \operatorname{Re} p_{n}(z)<\gamma$ for $\gamma>1$.

Corollary 3.2. $P_{n} \subset P, n \in N$.

Proof. Since $p_{0} \in P$, we have $\operatorname{Re} p_{0}(z)>0$. Therefore by Theorem 3.1 we have $\operatorname{Re}$ $p_{1}(z)>0$, and hence $\operatorname{Re} p_{2}(z)>0$ and so on for each $n \in N$.

Theorem 3.3. $P_{n+1} \subset P_{n}$

Proof. Let $p_{n+1}(z)$ belong to $P_{n+1}$. Then there exists $p \in P$ such that

$$
p_{n+1}(z)=\chi_{n+1}^{(\alpha)}(p(z))
$$

Then by Remark 1.2, $p_{n+1}(z)=\chi_{n}{ }^{(\alpha)}\left(\chi_{1}{ }^{(\alpha)}(p(z))\right)$, thus by Corollary $3.2 \chi_{1}{ }^{(\alpha)}(p(z))$ $=p_{1}(z)$ is a function in $P$, therefore $p_{n+1}(z)$ is the $n$th integral transform of a function in $P$, that is, $p_{n+1}(z)$ belongs to $P_{n}$. This completes the proof.

Corollary 3.4.([1]) Let $p \in P$ and $\gamma+c>0$. Then

$$
q(z)=1+(\gamma+c) \sum_{k=1}^{\infty} \frac{p_{k} z^{k}}{(\gamma+c+k)}, \quad z \in E
$$

is also in $P$.

The above corollary and its extension in [6] follow easily by taking $\alpha=\gamma+c>0$, $n=0$ in Theorem 3.3. The proofs in the two articles made use of a result of Miller and Mocanu [5, Theorem 10], which as observed in MR96j:30018, may not be applied directly except $\gamma+c$ is an integer.

Theorem 3.5. The transformation (1.3) is starlikeness-preserving. In other words, if $p \in P$ is starlike in $E$, then its transform $p_{n}$ is also starlike in $E$.

Proof. From (1.3) we get

$$
\frac{z p^{\prime}(z)}{p_{n}(z)}+\alpha=\frac{z^{\alpha} p_{n-1}(z)}{\int_{0}^{z} t^{\alpha-1} p_{n-1}(t) d t} \equiv \frac{M(z)}{N(z)} .
$$

Assume $p_{n-1}(z)$ is starlike in $E$. Then $N(z)$ is also starlike (in fact $N(z)$ maps $E$ onto a convex domain) since

$$
1+\frac{z N^{\prime \prime}(z)}{N^{\prime}(z)}=\alpha+\frac{z p_{n-1}^{\prime}(z)}{p_{n-1}(z)}
$$


Now from (3.6) let

$$
H(z)=\frac{z p_{n}^{\prime}(z)}{p_{n}(z)}=\frac{M(z)}{N(z)}-\alpha
$$

so that

$$
\frac{M^{\prime}(z)}{N^{\prime}(z)}-\alpha=H(z)+\frac{N(z)}{N^{\prime}(z)} H^{\prime}(z)=H(z)+\frac{z H^{\prime}(z)}{\eta(z)}
$$

where $\eta(z)=z N^{\prime}(z) / N(z)$. We write (3.9) as

$$
\frac{M^{\prime}(z)}{N^{\prime}(z)}-\alpha=\psi\left(H(z), z H^{\prime}(z)\right)
$$

where $\psi=\psi_{1}(u, v)=u+v / \eta, \operatorname{Re} \eta>0$ with $D=C \times C$ belongs to $\Psi$. But $M^{\prime}(z) / N^{\prime}(z)-$ $\alpha=z p_{n-1}{ }^{\prime}(z) / p_{n-1}(z)$. Thus we have $\operatorname{Re} \psi\left(H(z), z H^{\prime}(z)\right)>0$ which implies $\operatorname{Re} H(z)>$ 0 . That is, $\operatorname{Re} z p_{n}{ }^{\prime}(z) / p_{n}(z)>0$. Hence $p_{0}(z)$ is starlike in $E \Rightarrow p_{1}(z)$ is $\Rightarrow \cdots \Rightarrow p_{n}(z)$ is. This concludes the proof.

Corollary 3.6. The transformation $L_{n}(z)$ of the Moebius function is starlike and univalent in $E$.

Theorem 3.7. The transformation (1.3) is convexity-preserving. In other words, if $p \in P$ is convex in $E$, then its transform $p_{n}$ is also convex in $E$.

Proof. Observe from (1.3) that

$$
z p_{n}^{\prime}(z)=\frac{\alpha}{z^{\alpha}} \int_{0}^{z} t^{\alpha-1}\left(t p_{n-1}^{\prime}(t)\right) d t
$$

so that if $p_{n-1}(z)$ is convex in $E$, then $z p_{n-1}{ }^{\prime}(z)$ is starlike in $E$. Hence from (3.11) we conclude using Theorem 3.5 that $z p_{n}{ }^{\prime}(z)$ is starlike in $E$ and therefore $p_{n}(z)$ is convex in $E$. That is, $p_{0}(z)$ is convex in $E \Rightarrow p_{1}(z)$ is $\Rightarrow \cdots \Rightarrow p_{n}(z)$ is, and the proof is concluded.

Corollary 3.8. The transformation $L_{n}(z)$ of the Moebius function is convex in $E$.

Theorem 3.9. Let $p_{n} \in P_{n}$. Then

$$
\begin{aligned}
& \left|p_{n}(z)\right| \leq 1+2 \sum_{k=1}^{\infty} \frac{\alpha^{n}}{(\alpha+k)^{n}} r^{k}, \quad|z|=r \\
& \operatorname{Re} p_{n}(z) \geq 1+2 \sum_{k=1}^{\infty} \frac{\alpha^{n}}{(\alpha+k)^{n}}(-r)^{k}, \quad|z|=r .
\end{aligned}
$$

The results are sharp.

Proof. The transform $p_{n}(z)$ admits the representation (1.4). Thus by triangle inequality, using (1.6) we have the upper bound (3.12). Equality is realized for the function $p_{n}(z)=L_{n}(z)$. 
For the lower bound we use (1.3) where

$$
\operatorname{Re} p_{1}(z)=\operatorname{Re} \frac{\alpha}{z^{\alpha}} \int_{0}^{z} t^{\alpha-1} p(t) d t .
$$

Let $z=r e^{i \theta}$ and $t=\rho e^{i \theta}, 0<\rho \leq r<1$. Then (3.14) gives

$$
\operatorname{Re} p_{1}\left(r e^{i \theta}\right)=\operatorname{Re} \frac{\alpha}{r^{\alpha}} \int_{0}^{r} \rho^{\alpha-1} p\left(\rho e^{i \theta}\right) d \rho
$$

which gives

$$
\operatorname{Re} p_{1}\left(r e^{i \theta}\right)=\frac{\alpha}{r^{\alpha}} \int_{0}^{r} \rho^{\alpha-1} \operatorname{Re} p\left(\rho e^{i \theta}\right) d \rho .
$$

Since $p \in P$, Re $p\left(r e^{i \theta}\right) \geq(1-r) /(1+r)$ so that (3.16) yields

$$
\operatorname{Re} p_{1}\left(r e^{i \theta}\right) \geq 1+2 \sum_{k=1}^{\infty} \frac{\alpha}{\alpha+k}(-r)^{k} .
$$

Next we assume that for $1 \leq j \leq n$,

$$
\operatorname{Re} p_{j}\left(r e^{i \theta}\right) \geq 1+2 \sum_{k=1}^{\infty} \frac{\alpha^{j}}{(\alpha+k)^{j}}(-r)^{k} .
$$

Then by letting $z=r e^{i \theta}$ and $t=\rho e^{i \theta}, 0<\rho \leq r<1$, we would have

$$
\operatorname{Re} p_{j+1}\left(r e^{i \theta}\right)=\operatorname{Re} \frac{\alpha}{r^{\alpha}} \int_{0}^{r} \rho^{\alpha-1} p_{j}\left(\rho e^{i \theta}\right) d \rho
$$

giving

$$
\operatorname{Re} p_{j+1}\left(r e^{i \theta}\right)=\frac{\alpha}{r^{\alpha}} \int_{0}^{r} \rho^{\alpha-1} \operatorname{Re} p_{j}\left(\rho e^{i \theta}\right) d \rho .
$$

Using (3.18) in (3.20) we get

$$
\operatorname{Re} p_{j+1}\left(r e^{i \theta}\right) \geq 1+2 \sum_{k=1}^{\infty} \frac{\alpha^{j+1}}{(\alpha+k)^{j+1}}(-r)^{k} .
$$

Therefore the inequality (3.13) follows by induction. Equality is attained for $p_{n}(z)=$ $L_{n}(-z)$.

It is well known that if an analytic function $g(z)$ is univalent in $E$, then $f(z)$ is subordinate to $g(z)$ (written as $f \prec g$ ) if and only if $f(E) \subset g(E)$ and $f(0)=g(0)$ [7]. Therefore the following corollary follows from Corollary 3.6 and Theorem 3.9.

Corollary 3.10. $p_{n} \in P_{n}$ if and only if $p_{n}(z) \prec L_{n}(z)$.

Remark 3.11. If we choose $n=0$ in the corollary above we see that $p \in P$ if and only if $p(z) \prec L_{0}(z)$ which is well known. 
Finally from Definition 1.1, Corollary 3.10 and other well-known facts about the class $P$, we have the following equivalence:

Remark 3.12. For $z \in E$, the following are equivalent:

(i) $p \prec L_{0}(z)$

(ii) $p \in P$

(iii) $p_{n} \in P_{n}$

(iv) $p_{n} \prec L_{n}(z)$.

The above equivalence relation shows that the iterated integral transformations (1.3) of functions in the family $P$ of Caratheodory functions not only preserve starlikeness and convexity but also subordination-preserving. Thus the resulting infinite sequence $\left\{d_{k}\right\}_{1}^{\infty}=\frac{\alpha^{n}}{(\alpha+k)^{n}}, n \in N_{0}$ (see equation (1.5)) has the property that it is preserving of many geometric structures of the family $P$. The important role of such sequence in geometric function theory has been studied by Bernardi in [2].

\section{Applications}

The family $P_{n}$ actually arose from the study of classes $B_{n}(\alpha)$ and $T_{n}^{\alpha}(\beta)$ introduced in $[1,6]$ and has proved very resourceful in dealing easily with certain problems of the theory of analytic and univalent functions as will be demonstrated shortly. First, we recall that a function $f(z)$ defined by $(1.2)$ is said to belong to $B_{n}(\alpha)$ if and only if $\operatorname{Re}$ $\left\{D^{n} f(z)^{\alpha} / z^{\alpha}\right\}>0, \alpha>0$ is real, $D^{n}\left(n \in N_{0}=\{0,1,2, \ldots\}\right)$ is the Salagean derivative defined by the relations $D^{0} f(z)=f(z)$ and $D^{n} f(z)=z\left[D^{n-1} f(z)\right]^{\prime}[1,6,8]$. The class $T_{n}^{\alpha}(\beta)$ was defined in [6] as an extension of $B_{n}(\alpha)$, however certain errors have been pointed out in the work (see MR96j: 30018). We acknowledge those errors and assert that they are due to a misstatement of the associated geometric condition. Thus we say:

Definition 4.1. A normalized analytic function given by (1.2) belongs to the class $T_{n}^{\alpha}(\beta)$ if and only if

$$
\operatorname{Re} \frac{D^{n} f(z)^{\alpha}}{\alpha^{n} z^{\alpha}}>\beta
$$

where $0 \leq \beta<1$ and $\alpha$ and $D^{n}$ are as already defined.

In this section we will present several different applications of the transformation $P_{n}$, mainly in the study of functions in the class $T_{n}^{\alpha}(\beta)$, both to obtain new results and to provide very simple proofs for some known ones. Several choices of the parameters $n, \alpha$ and $\beta$ lead to corresponding results in classes $B_{n}(\alpha), B_{1}(\alpha), R$ and $S_{0}[1,4,9,10]$.

The following lemma gives the basic relationship between the classes $P_{n}$ and $T_{n}^{\alpha}(\beta)$.

Lemma 4.2. Let $f(z)$ be given by (1.2), and $\alpha, \beta$ and $D^{n}$ as defined above. Then the following are equivalent:

(i) $f \in T_{n}^{\alpha}(\beta)$ 
(ii) $\left(D^{n} f(z)^{\alpha} / \alpha^{n} z^{\alpha}-\beta\right) /(1-\beta) \in P$

(iii) $\left(f(z)^{\alpha} / z^{\alpha}-\beta\right) /(1-\beta) \in P_{n}$.

Proof. That (i) $\Leftrightarrow$ (ii) is clear from Definition 4.1. Now (ii) is true $\Leftrightarrow$ there exist $p \in P$ such that

$$
D^{n} f(z)^{\alpha}=\alpha^{n} z^{\alpha}(\beta+(1-\beta) p(z))
$$

We shall apply on (4.1) the integral operator, $I_{n}$, defined in [8] as

$$
I_{n} f(z)=I\left(I_{n-1} f(z)\right)=\int_{0}^{z} \frac{I_{n-1} f(t)}{t} d t,
$$

with $I_{0} f(z)=f(z)$ so that we have equation $(4.1) \Leftrightarrow$

$$
f(z)^{\alpha}=z^{\alpha}+(1-\beta) \sum_{k=1}^{\infty} \frac{\alpha^{n}}{(\alpha+k)^{n}} p_{k} z^{\alpha+k}
$$

$\Leftrightarrow$

$$
\frac{f(z)^{\alpha} / z^{\alpha}-\beta}{1-\beta}=1+\sum_{k=1}^{\infty} \frac{\alpha^{n}}{(\alpha+k)^{n}} p_{k} z^{k}
$$

which proves the lemma.

Now we are in position to give new proofs of some earlier results in $[1,6]$ and obtain further characterization of the class $T_{n}{ }^{\alpha}(\beta)$.

Theorem 4.3. $T_{n+1}{ }^{\alpha}(\beta) \subset T_{n}{ }^{\alpha}(\beta)$.

Proof. Let $f \in T_{n+1}{ }^{\alpha}(\beta)$. Then by Lemma $4.2\left(f(z)^{\alpha} / z^{\alpha}-\beta\right) /(1-\beta) \in P_{n+1}$. By Theorem $3.3\left(f(z)^{\alpha} / z^{\alpha}-\beta\right) /(1-\beta) \in P_{n}$. That is $f \in T_{n}{ }^{\alpha}(\beta)$.

Corollary 4.4. For $n \geq 1, T_{n}{ }^{\alpha}(\beta) \subset S$ (the class of functions $f(z)$ given by $(1.2)$ which are univalent in $E$ ).

Theorem 4.5. Let $f(z)$ given by $(1.2)$ be in the class $T_{n}{ }^{\alpha}(\beta)$. Then the function $F(z)$ defined by

$$
F(z)^{\alpha}=\frac{\alpha+c}{z^{c}} \int_{0}^{z} t^{c-1} f(t)^{\alpha} d t, \quad \alpha+c>0
$$

is also in $T_{n}{ }^{\alpha}(\beta)$.

Proof. From (4.4) we have

$$
\frac{F(z)^{\alpha} / z^{\alpha}-\beta}{1-\beta}=\frac{v}{z^{v}} \int_{0}^{z} t^{v-1}\left(\frac{f(t)^{\alpha} / t^{\alpha}-\beta}{1-\beta}\right) d t
$$

where $v=a+c$. Since $f \in T_{n}{ }^{\alpha}(\beta)$, using Remark 1.2 and Lemma 4.2, we write (4.5) as

$$
\frac{F(z)^{\alpha} / z^{\alpha}-\beta}{1-\beta}=\chi_{1}{ }^{(v)}\left(\chi_{n}{ }^{(\alpha)}(p(z))\right)=\chi_{n}{ }^{(\alpha)}\left(\chi_{1}{ }^{(v)}(p(z))\right) \text {. }
$$


Equation (4.6) implies that $\left(F(z)^{\alpha} / z^{\alpha}-\beta\right) /(1-\beta)$ belong to the class $P_{n}$. Therefore by Lemma $4.2, F \in T_{n}{ }^{\alpha}(\beta)$.

Theorem 4.6. A function $F(z)$ defined by $F(z)^{\alpha+v}=z^{v} f(z)^{\alpha}$ (where $f(z)$ is given by (1.2)) is in the class $T_{n}{ }^{\alpha+v}(\beta)$ if and only if $f(z)$ is in $T_{n}{ }^{\alpha}(\beta)$.

Proof. By definition of $F(z)$

$$
\frac{F(z)^{\alpha+v}}{z^{\alpha+v}} \equiv \frac{f(z)^{\alpha}}{z^{\alpha}}
$$

and the result follows (cf. [9]).

Theorem 4.7. Let $f \in T_{n}{ }^{\alpha}(\beta)$ and define

$$
M_{T}(n, \alpha, \beta, r)=r\left\{1+2(1-\beta) \alpha^{n} \sum_{k=1}^{\infty} \frac{r^{k}}{(\alpha+k)^{n}}\right\}^{\frac{1}{\alpha}}
$$

and

$$
m_{T}(n, \alpha, \beta, r)=r\left\{1+2(1-\beta) \alpha^{n} \sum_{k=1}^{\infty} \frac{(-r)^{k}}{(\alpha+k)^{n}}\right\}^{\frac{1}{\alpha}} .
$$

Then $m_{T}(n, \alpha, \beta, r) \leq|f(z)| \leq M_{T}(n, \alpha, \beta, r)$. The inequalities are sharp.

Proof. The result follows by taking $p_{n}(z)=\left(f(z)^{\alpha} / z^{\alpha}-\beta\right) /(1-\beta)$ in Theorem 3.9. Equality in the upper bound is realized for the functions $f(z)$ given by

$$
\frac{D^{n} f(z)^{\alpha}}{\alpha^{n} z^{\alpha}}=\frac{1+(1-2 \beta) z}{1-z}
$$

while equality in the lower bound is attained by the functions $f(z)$ given by

$$
\frac{D^{n} f(z)^{\alpha}}{\alpha^{n} z^{\alpha}}=\frac{1-(1-2 \beta) z}{1-z} .
$$

This completes the proof.

Theorem 4.8. Each function $f(z)$ in the class $T_{n}{ }^{\alpha}(\beta)$ maps the unit disk onto a domain which covers the disk $|\xi|<m_{T}(n, \alpha, \beta, 1)$. The result is sharp.

Proof. From Theorem 4.7, we have $|f(z)| \geq m_{T}(n, \alpha, \beta, r)$. This implies that range of every function $f(z)$ in the class covers the disk $|w|<m_{T}(n, \alpha, \beta, 1)=\inf _{r \rightarrow 1} m_{T}(n, \alpha, \beta, r)$. The functions $f(z)$ given by (4.9) show that the result is sharp.

Theorem 4.9. Let $f \in T_{n}{ }^{\alpha}(\beta)$ and define

$$
M_{T}^{*}(n, \alpha, \beta, r)=r^{\alpha-1}\left\{1+2(1-\beta) \sum_{k=1}^{\infty} \frac{\alpha^{n-1}}{(\alpha+k)^{n-1}} r^{k}\right\}
$$


and

$$
m_{T}^{*}(n, \alpha, \beta, r)=r^{\alpha-1}\left\{1+2(1-\beta) \sum_{k=1}^{\infty} \frac{\alpha^{n-1}}{(\alpha+k)^{n-1}}(-r)^{k}\right\} .
$$

Then $m^{*} T(n, \alpha, \beta, r) \leq\left|f(z)^{\alpha-1} f^{\prime}(z)\right| \leq M^{*}{ }_{T}(n, \alpha, \beta, r)$. The inequalities are sharp.

Proof. Since $f \in T_{n}{ }^{\alpha}(\beta)$, by Lemma 4.2 , there exists $p_{n} \in P_{n}$ such that

$$
f(z)^{\alpha}=z^{\alpha}\left[\beta+(1-\beta) p_{n}(z)\right] .
$$

Hence we have

$$
\frac{f(z)^{\alpha-1} f^{\prime}(z)}{z^{\alpha-1}}=\beta+(1-\beta)\left[p_{n}(z)+z p_{n}^{\prime}(z) / \alpha\right] .
$$

From (1.3) we find that $p_{n}(z)+z p_{n}^{\prime}(z) / \alpha=p_{n-1}(z)$ so that (4.11) becomes

$$
\frac{f(z)^{\alpha-1} f^{\prime}(z)}{z^{\alpha-1}}=\beta+(1-\beta) p_{n-1}(z) .
$$

Using Theorem 3.9 we get

$$
\left|\frac{f(z)^{\alpha-1} f^{\prime}(z)}{z^{\alpha-1}}\right| \leq 1+2(1-\beta) \sum_{k=1}^{\infty} \frac{\alpha^{n-1}}{(\alpha+k)^{n-1}} r^{k}
$$

and

$$
\operatorname{Re} \frac{f(z)^{\alpha-1} f^{\prime}(z)}{z^{\alpha-1}} \geq 1+2(1-\beta) \sum_{k=1}^{\infty} \frac{\alpha^{n-1}}{(\alpha+k)^{n-1}}(-r)^{k} .
$$

The inequalities now follow from (4.13) and (4.14). Upper bound equality is realized for the functions $f(z)$ given by (4.8) while in the lower bound equality is attained by the functions $f(z)$ defined by (4.9).

Finally we note that the integral of Abdulhalim [1, Theorem 3.5] can be written as $I_{m}(z)=\chi_{m}{ }^{(v)}\left(\chi_{n}{ }^{(\alpha)}(p(z))\right)$ where $\alpha>0, v=\alpha+1>0, m, n \in N_{0}$ and $\chi_{n}{ }^{(\alpha)}(p(z))=$ $I_{0}(z)=f(z)^{\alpha} / z^{\alpha}$ so that his result follows by Remark 1.2 and Theorem 3.9. Extension to $T_{n}{ }^{\alpha}(\beta)$ follows by taking $\chi_{n}{ }^{(\alpha)}(p(z))=\left(f(z)^{\alpha} / z^{\alpha}-\beta\right) /(1-\beta)$.

\section{Acknowledgement}

The first author acknowledges with many thanks the tremendous assistance offered him by Prof. Micheal Dorff, Brigham Young University, Provo, Utah, USA during his (first author's) doctoral work at the University of Ilorin, Nigeria, leading to this and other publications. 


\section{References}

[1] S. Abdulhalim, On a class of analytic functions involving the Salagean differential operator, Tamkang J. Maths. 23 (1992), 51-58.

[2] S. D. Bernard, Convex and starlike functions, Trans. Amer. Math. Soc. 135 (1969), 429446.

[3] Z. Lewandowski, S. Miller and E. Zlotkiewicz, Generating functions for some classes of univalent functions, Proc. Amer. Math. Soc. 56 (1976), 111-117.

[4] T. H. Macgregor, Function whose derivatives have positive real part, Trans. Amer. Math. Soc. 104 (1962), 532-537. MR 25-797.

[5] S. S. Miller and P. T. Mocanu, Second order differential inequalities in the complex plane, J. Math. Anal. Appl. 65 (1978), 289-305.

[6] T. O. Opoola, On a new subclass of univalent function, Matematika Tome (36) 59(2) (1994), 195-200.

[7] Ch. Pommerenke, Univalent Functions. Springer-Verlag. New York Inc. 1983.

[8] G. S. Salagean, Subclasses of univalent functions, Lecture Notes in Math. 1013 (1983), 362-372. Springer-Verlag, Berlin, Heidelberg \& New York.

[9] R. Singh, On Bazilevic functions, Proc. Amer. Math. Soc. 38 (1973), 261-271. MR 47 \# 449.

[10] K. Yamaguchi, On functions satisfying $\operatorname{Re}\{f(z) / z\}>0$, Proc. Amer. Math. Soc. 17 (1966), 588-591. MR 33 \# 268.

Department of Mathematics, University of Ilorin, Ilorin, Nigeria.

E-mail: abuqudduus@yahoo.com

Department of Mathematics, University of Ilorin, Ilorin, Nigeria.

E-mail: opoola_stc@yahoo.com 